\title{
Special Issue on Variational Inequalities: Consistent Conjectural Variations Coincide with the Nash Solution in the Meta-Model
}

\author{
Viacheslav Kalashnikov ${ }^{1,2,3}$ (D) . Nataliya Kalashnykova ${ }^{4}$ José G. Flores-Muñiz ${ }^{4}$
}

Published online: 7 August 2019

(C) The Author(s) 2019

\begin{abstract}
We keep investigating the properties of consistent conjectural variations equilibrium (CCVE) developed for a single-commodity oligopoly. Although, in general, the consistent conjectures are distinct from those of Cournot-Nash, in our previous papers, we established the following remarkable fact. Define a meta-model as such where the players are the same agents as in the original oligopoly but now using the conjectures as their strategies. Then the Cournot-Nash equilibrium in the meta-model generated the consistent conjectural variations equilibrium in the original oligopoly. In this paper, we study the conditions under which the inverse is also true, that it, every consistent CVE provides for the Cournot-Nash optimal strategies for the metamodel. This equivalence allows one to extend the concept of CCVE to other kinds of economic and financial models lacking the oligopoly structure.
\end{abstract}

Keywords Consistent conjectural variations equilibrium · Meta-model · Optimal Cournot-Nash strategies

\section{Introduction}

The concept of conjectural variations equilibrium (CVE) was first proposed by Bowley (1924) and Frisch (1933) in order to extend the concept of a solution to a static (Cournot) model (game). According to this concept, the game players behave in the following manner: each agent chooses her/his most profitable strategy remembering that each adversary's action is a conjectured function of her/his own strategy.

This research was financially supported by the Mexico SEP-CONACYT grants CB-2013-01-221676 and FC-2016-01-1938.

Viacheslav Kalashnikov

kalash@tec.mx

Extended author information available on the last page of the article. 
The papers (Bulavsky and Kalashnikov 1994; 1995) and the monograph (Isac et al. 2002) introduce and examine a new form of the CVE, in which the conjectural variations (represented via the influence coefficients of each agent) were used to bring about a new equilibrium concept distinct from that of Cournot-Nash.

For instance, in Isac et al. (2002), the classical oligopoly model was extended to the conjectural oligopoly as follows. Instead of the usual Cournot-Nash assumptions, all producers $i=1, \ldots, n$, considered the conjectural variations described below:

$$
G_{i}(\eta)=G+\left(\eta-q_{i}\right) w_{i}\left(G, q_{i}\right) .
$$

Here, $G$ is the current total quantity of the product cleared in the market, $q_{i}$ and $\eta$ are, respectively, the present and the expected supplies by the $i$-th agent, whereas $G_{i}(\eta)$ is the total cleared market volume conjectured by the $i$-th agent as a response to changing her own supply from $q_{i}$ towards $\eta$. The conjecture function $w_{i}$ was referred to as the $i$-th agent's influence quotient (coefficient). Recall that the usual Cournot-Nash model assumes $w_{i} \equiv 1$ for all $i, i=1, \ldots, n$. Under general enough assumptions concerning the properties of the influence coefficients $w_{i}=w_{i}\left(G, q_{i}\right)$, cost functions $f_{i}\left(q_{i}\right)$, and the inverse demand function (or, price function) $p=p(G)$, new existence and uniqueness results for the conjectural variations equilibrium (CVE) were obtained. This approach was further developed in Kalashnikov et al. (2009, 2010).

An interesting comparison of the Cournot-Nash and Bertran models is conveyed in Kreps and Scheinkman (1983). Bertrand's model of oligopoly, which considers the perfect competition, assumes that (1) there is a competition over prices and (2) production follows the realization of the demand. The authors (Kreps and Scheinkman 1983) demonstrate that both of these assumptions are required. In more detail, they study a two-stage oligopoly game where, first, there is a simultaneous production, and, second, after the production levels are made public, there is price competition. Under rather mild assumptions about the demand, the authors show in Kreps and Scheinkman (1983) that the unique equilibrium is the Cournot-Nash one. This illustrates that solutions to oligopoly games depend on both the strategic variables employed and the context (game form) in which those variables are employed.

A different example of a two-stage game can be seen in Murphy and Smeers (2005). Similar studies kept going in the unpublished manuscript (Kimbrough et al. 2014) dealing mainly with forward markets but still providing important insight into the consistent conjectural variations equilibrium in the many-stage oligopoly model. The authors examine the impact of the conjectures about the players' knowledge on the outcome of the game, where the outcomes are consistent with their conjectures. Then they deduce the similar result obtained by Allaz and Vila (1993) on forward markets but under different assumptions of knowledge from consistent conjectural variations.

All the above-mentioned papers deal in various modes with games with conjectural variations equilibrium (CVE). A more detailed story of the "highs and lows" of the CVE can be found in Kalashnikov et al. $(2011,2017)$. However, for the sake of a complete list of the predecessors, we briefly repeat the short survey presented in Kalashnikov et al. (2017). 
As is mentioned in Figuières et al. (2004), Giocoli (2005), the concept of conjectural variations has been the subject of numerous theoretical controversies (e.g., see Lindh (1992)). Nevertheless, economists have made extensive use of one form or the other of the CVE to predict the outcome of non-cooperative behavior in several fields of economics. The literature on conjectural variations has focused mainly on two-player games ( $c f$., Figuières et al. (2004)). The central concept of the theory is the notion of conjecture. Usually, the variational conjecture describes the reaction of player $j$, as anticipated by player $i$, to an infinitesimal variation of player $i$ 's strategy. This mechanism leads to the notion of a conjectured reaction function of the opponent. Given these conjectured reactions on the part of the rivals, each agent optimizes his perceived payoff. This yields the concept of a conjectural best response function. Equilibrium is obtained when no player has an interest in deviating from his/her strategy, i.e., his/her conjectural best response to the strategies of the other player. Here, it is worthwhile to recall again that we are studying the static equilibrium where conjectures, prices, and production volumes are selected simultaneously by applying the consistency criterion.

The main obstacle in the way of admitting this concept is a problem with its consistency. The consistency (a.k.a., sometimes, "rationality") of the equilibrium is defined as the coincidence of the conjectural best response by each agent and the conjectured reaction function of the same agent. A conceptual difficulty arises when one considers consistency in the case of many (i.e., more than 2) agents (see, again, Kalashnikov et al. (2017) and Figuières et al. (2004)). Interesting discussions of the concept of consistency of predictions can be also found in the papers Ben-Akiva et al. (2001) and Zerrahn and Huppmann (2017).

Indeed, the strongest notion of consistency requires that the conjectural best response of player $i$ coincides with what the other players have conjectured about her, that is, with one of their conjectured reaction functions. However, when $n$ agents are present, there are $n$ best response functions and $n(n-1)$ conjectures. Therefore, if $n>2$, an equilibrium is consistent only if all players have the same conjectures about player $i$. This is the approach followed explicitly by Başar and Olsder (1982); this assumption can be also found in Fershtman and Kamien (1987) dealing with conjectures in differential games. In the literature on static $n$-player conjectural variations, the problem is usually implicitly addressed by assuming a complete identity of all the agents (cf., Laitner (1980), Bresnahan (1981), and references therein; Novshek (1985)). Using a bit different approach, Perry (1982) for oligopoly, Cornes and Sandler (1984) and Sugden (1985) for public goods, consider a class of games where for each agent, the contributions of all other players to her payoff are aggregated. It is as if each agent plays against a unique (virtual) player representing the remaining agents.

In order to cope with such a conceptual difficulty arising in many-player games, a completely new approach was proposed in Bulavsky (1997). Instead of assuming the identity of the agents in the conjectural variation model of a homogeneous good market, it is supposed that each player makes conjectures not about the (optimal) response functions of the other players but only about first-order variations of the market price depending upon his/her infinitesimal output variations. Knowing the adversaries' conjectures (called influence coefficients), each agent can apply a verification procedure and check if his/her influence coefficient is consistent (compatible) 
with those of the rest of the agents. Exactly the same verification formulas were obtained independently (but 10 years later) in Liu et al. (2007) establishing the existence and uniqueness of consistent conjectural variation equilibrium in an electricity market. However, to do that, the authors of Liu et al. (2007) made use of a much more complicated optimal control technique when searching the system's steady states (a similar approach was handled in Driskill and McCafferty (1989)). Moreover, in Liu et al. (2007), the inverse demand function is linear, and the agents' cost functions are quadratic, whereas Bulavsky (1997) allows nonlinear and even non-differentiable demand functions as well as arbitrary convex cost functions of the agents.

The results obtained in Bulavsky (1997) for classical oligopoly models were extended in Kalashnikov et al. (2011) to a mixed oligopoly model. In the same manner as in Bulavsky and Kalashnikov (1994, 1995), the authors considered a conjectural variations oligopoly model, in which the degree of influence upon the general situation by each agent is modeled by special parameters (influence coefficients). However, in contrast to the models defined in Bulavsky and Kalashnikov (1994, 1995) and Kalashnikov et al. (2009, 2010), the papers Kalashnikov et al. (2011) and Kalashnikov et al. (2017) follows the pattern proposed in Bulavsky (1997) selecting the market clearing price $p$, rather than the producers' total output $G$, as an observable variable. Because of that, the players' conjectures are denoted by letters $v_{i}$ instead of the previously employed $w_{i}$, with an evident relationship valid for these pairs of parameters:

$$
w_{i}=-\frac{v_{i}}{p^{\prime}(G)}, \quad i=1, \ldots, n .
$$

As was demonstrated in Kalashnikov et al. (2011), in general, the consistent conjectures (in terms of $w_{i}$ ) are not the Cournot-Nash conjectures, i.e., $w_{i} \neq 1$. In other words, at a consistent CVE, each player $i$ does not use the Cournot-Nash equilibrium concept, since he/she doesn't assume that all other $(j \neq i)$ agents are stuck to the (equilibrium) production volumes $q_{j}, j \neq i$. In our previous paper (Kalashnikov et al. 2017), we introduced a meta-model, in which not the players' production volumes $q_{i}$ but their conjectures $v_{i}$ serve as the players' strategies instead. The remarkable fact was demonstrated: the consistent (for the original oligopoly) conjectures $v_{i}^{*}$, while in general not being the Cournot-Nash ones for this game, are the optimal Cournot-Nash strategies in the above-mentioned meta-model. In other words, if each player $i$ assumes that the rest of the players stick to their consistent CVE conjectures $v_{j}^{*}, j \neq i$ then his/her consistent conjecture $v_{i}^{*}$ is optimal for player $i$, too. The latter means that the vector of (consistent in the original oligopoly) conjectures $v_{i}^{*}, i=1, \ldots, n$, coincides with the classical Cournot-Nash equilibrium in the meta-game. Similar results were claimed (without proof) in our previous paper (Kalashnykova et al. 2012) but only for quadratic cost functions.

However, since the meta-model allows the agents to select their strategies from $R^{n}$, and the latter isn't compact, the existence of the Cournot-Nash equilibrium in the meta-model has to be guaranteed by some extra assumptions (similar difficulties related to the price equilibrium were run into and overcome by Kress and Pesch (2015)). Under those assumptions, the complete equivalence of the consistent CVE in the original oligopoly and the meta-model has been established in this paper. 
The rest of the paper is organized as follows. In Section 2, the mathematical model from Bulavsky (1997) is described and then, in Section 3, the concept of exterior equilibrium (i.e., the conjectural variations equilibrium (CVE) with the influence coefficients fixed in an exogenous form) is defined. The existence and uniqueness theorems for this kind of CVE end the section. Section 4 deals with a more advanced concept of interior equilibrium, which is determined as the exterior equilibrium with consistent conjectures (influence coefficients). The consistency criterion, its verification procedure, and the existence theorems for the interior equilibrium are formulated in the same Section 4. Even though the short Sections 2 to 4 mainly are the same as in our recent paper (Kalashnikov et al. 2017), we insert them to make this paper somewhat self-contained. Finally, Section 5 establishes the main (and the only new) results of this manuscript asserting that the consistent conjectural equilibrium in the original oligopoly provides the classical Cournot-Nash equilibrium in the meta-model and vice versa. The main body of the paper is finished with Section 6 that presents a couple of numerical examples that illustrate the applicability of the obtained results to real cases. The proofs of the three principal theorems of this paper are exported to the Appendix.

\section{Model Specification}

Consider an oligopoly of at least two producers of a homogeneous good with the cost functions $f_{i}=f_{i}\left(q_{i}\right), i \in\{1, \ldots, n\}, n \geq 2$, where $q_{i} \geq 0$ is the supply by producer $i$. Consumers' demand is described by a demand function $G=G(p)$, whose argument $p$ is the market price established by a cleared market. An active demand $D$ is nonnegative and does not depend upon the price. The equilibrium between the demand and supply for a given price $p$ is guaranteed by the following balance equality:

$$
\sum_{i=1}^{n} q_{i}=G(p)+D .
$$

We assume the following properties of the model's data:

A2.1 The demand function $G=G(p) \geq 0$ is defined for $p>0$, being decreasing and continuously differentiable.

A2.2 For each $i \in\{1, \ldots, n\}$, the function $f_{i}=f_{i}\left(q_{i}\right)$ is defined for every $q_{i} \geq 0$, is twice continuously differentiable, and in addition, the following inequalities hold:

$$
f_{i}^{\prime}(0)>0 \text { and } f_{i}^{\prime \prime}\left(q_{i}\right)>0, \forall q_{i} \geq 0 .
$$

Next, every producer $i \in\{1, \ldots, n\}$ chooses its output volume $q_{i} \geq 0$ so as to maximize its net profit function:

$$
\pi_{i}\left(p, q_{i}\right)=p q_{i}-f_{i}\left(q_{i}\right)
$$


Now we postulate that the producers admit that their perturbations in production volumes may affect the price value $p$. In first order terms, the latter assumption is implemented by means of a conjectural dependence of infinitesimal variations of the price $p$ upon infinitesimal variations of the supply volumes $q_{i}$. If so, the first order maximum condition to describe the equilibrium will have the form:

$$
\frac{\partial \pi_{i}}{\partial q_{i}}=p+q_{i} \frac{\partial p}{\partial q_{i}}-f_{i}^{\prime}\left(q_{i}\right)\left\{\begin{array}{l}
=0, \text { if } q_{i}>0, \\
\leq 0, \text { if } q_{i}=0,
\end{array} \quad i \in\{1, \ldots, n\} .\right.
$$

Therefore, to describe the (infinitesimal) behavior of producer $i$, it is enough to conjecture the first order derivative $\partial p / \partial q_{i} \equiv-v_{i}$ instead of the exact functional dependence of $p$ upon $q_{i}$. Here, we introduce the negative sign in order to deal with nonnegative values of $v_{i}$. Of course, the conjectured first-order dependence of $p$ on $q_{i}$ must provide, at least locally, the concavity of the $i$-th producer's conjectured profit as a function of its output (otherwise, one cannot guarantee the profit to be maximized). As we suppose that the cost functions $f_{i}$ are strictly convex and strictly increasing, by inequalities (4), then, for all $i \in\{1, \ldots, n\}$, the concavity of the product $p q_{i}$ with respect to $q_{i}$ would suffice. For instance, it is enough to assume the coefficient $v_{i}$ (from now on referred to as the $i$-th producer's influence coefficient) to be nonnegative and constant. Then, the conjectured (infinitesimal first order) dependence of the profit's variations upon the production output $\eta_{i}$ has the form:

$$
\widehat{\pi}_{i}\left(\eta_{i}\right)=\left[p-v_{i}\left(\eta_{i}-q_{i}\right)\right] \eta_{i}-f_{i}\left(\eta_{i}\right),
$$

which is a concave function on $\eta_{i}$.

Here, it is worthwhile to mention that relation (7) does not mean that agent $i$ exercises its market power, which would look weird as combined with the balance equality (3). In fact, vice versa, player $i$ is a price-taker: she/he accepts its conjectured influence coefficient $v_{i}$ and calculates the expected variation in the market clearing price $p$ (and hence in her/his net profit) under the infinitesimal variation of her/his produce $\left(\eta-q_{i}\right)$.

Therefore, the maximum necessary condition at $\eta_{i}=q_{i}$ is provided by the relationships

$$
\left\{\begin{array}{ll}
p=v_{i} q_{i}+f_{i}^{\prime}\left(q_{i}\right), & \text { if } q_{i}>0, \\
p \leq f_{i}^{\prime}(0), & \text { if } q_{i}=0,
\end{array} \quad i \in\{1, \ldots, n\},\right.
$$

and it is the sufficient condition, too.

If the producers' conjectures about the model are given in an exogenous mode like it was assumed in Bulavsky and Kalashnikov (1994, 1995), we could allow the values $v_{i}$ to be functions of $q_{i}$ and $p$. However, here we use the approach from papers (Kalashnykova et al. 2012) and (Bulavsky 1997), where the conjectured parameters for the equilibrium are determined simultaneously with the price $p$ and the output values $q_{i}$ by a special verification procedure. In the latter case, the influence coefficients are scalar parameters determined only for the equilibrium state. In what follows, such equilibrium state is referred as to interior, and it is described by the combined vector of variables and parameters $\left(p ; q_{1}, \ldots, q_{n} ; v_{1}, \ldots, v_{n}\right)$. Nevertheless, in order to 
present the verification procedure, we need first to introduce a simpler notion of equilibrium called exterior (cf., Bulavsky (1997)) with the parameters $v_{i}$ assigned in the exogenous form. This concept is defined and discussed in the next section.

\section{Exterior Equilibrium}

We define the concept of exterior equilibrium as follows:

Definition 3.1 A vector $\left(p ; q_{1}, \ldots q_{n}\right)$ is called exterior equilibrium for the given influence coefficients $v_{i} \geq 0, i \in\{1, \ldots, n\}$, if the market is balanced, i.e., equality (3) holds, and for each $i \in\{1, \ldots, n\}$, the maximum conditions (8) are valid.

From now onward, we are going to consider only the case when the set of really producing participants is fixed (i.e, it doesn't depend upon the values $v_{i}$ of the influence coefficients). To guarantee this feature, we make the assumption listed below.

A3.1 For $p_{0}=\max _{1 \leq i \leq n}\left\{f_{i}^{\prime}(0)\right\}$ and any $i \in\{1, \ldots, n\}$, there exists a unique (due to A2.2) supply volume $q_{i}^{0} \geq 0$ such that

$$
p_{0}=f_{i}^{\prime}\left(q_{i}^{0}\right), \text { and in addition, } \sum_{i=1}^{n} q_{i}^{0}<G\left(p_{0}\right) .
$$

Lemma 3.2 Assumptions A2.1, A2.2 and A3.1 imply that for all nonnegative values of $v_{i}, i \in\{1, \ldots, n\}$, any exterior equilibrium has its supply values $q_{i}$ strictly positive (that is, $\left.q_{i}>0, i \in\{1, \ldots, n\}\right)$ if, and only if $p>p_{0}$.

Proof cf., Kalashnikov et al. (2017) .

The existence and uniqueness of the exterior equilibrium for any set of (nonnegative) conjectures (influence coefficients) were established in Bulavsky (1997). However, in the latter paper, only differentiability of the equilibrium clearing price $p$ with respect to the active demand $D$ was proven, while in this paper, we also need to show that the same equilibrium price function $p=p\left(D, v_{1}, \ldots, v_{n}\right)$ is differentiable by the influence coefficients, too. Therefore, the following theorem has been proved.

Theorem 3.3 Under assumptions A2.1, A2.2 and A3.1, for any $D \geq 0, v_{i} \geq 0, i \in$ $\{1, \ldots, n\}$, there exists uniquely the exterior equilibrium $\left(p ; q_{1}, \ldots, q_{n}\right)$ that depends continuously on the parameters $D \geq 0, v_{i} \geq 0, i \in\{1, \ldots, n\}$. The equilibrium price $p=p\left(D, v_{1}, \ldots, v_{n}\right)$, as a function of these parameters, is differentiable with respect to both $D$ and $v_{i}, i \in\{1, \ldots, n\}$. Moreover, $p\left(D, v_{1}, \ldots, v_{n}\right)>p_{0}$, and

$$
\frac{\partial p}{\partial D}=\frac{1}{\sum_{i=1}^{n} \frac{1}{v_{i}+f_{i}^{\prime \prime}\left(q_{i}\right)}-G^{\prime}(p)},
$$


while

$$
\frac{\partial p}{\partial v_{i}}=\frac{\frac{q_{i}}{v_{i}+f_{i}^{\prime \prime}\left(q_{i}\right)}}{\sum_{k=1}^{n} \frac{1}{v_{k}+f_{k}^{\prime \prime}\left(q_{k}\right)}-G^{\prime}(p)}>0, i \in\{1, \ldots, n\}
$$

Similarly, the equilibrium supply $q_{i}=q_{i}\left(D, v_{1}, \ldots, v_{n}\right), i \in\{1, \ldots, n\}$, is differentiable with respect to the influence coefficients $v_{k}, k \in\{1, \ldots, n\}$, with the partial derivatives having the forms:

$$
\frac{\partial q_{i}}{\partial v_{i}}=-\frac{q_{i}}{v_{i}+f_{i}^{\prime \prime}\left(q_{i}\right)}\left[\begin{array}{l}
\sum_{\substack{k=1 \\
k \neq i}}^{n} \frac{1}{v_{k}+f_{k}^{\prime \prime}\left(q_{k}\right)}-G^{\prime}(p) \\
\sum_{k=1}^{n} \frac{1}{v_{k}+f_{k}^{\prime \prime}\left(q_{k}\right)}-G^{\prime}(p)
\end{array}\right]<0, i \in\{1, \ldots, n\},
$$

and

$$
\frac{\partial q_{i}}{\partial v_{j}}=\frac{1}{v_{i}+f_{i}^{\prime \prime}\left(q_{i}\right)}\left[\frac{\frac{q_{j}}{v_{j}+f_{j}^{\prime \prime}\left(q_{j}\right)}}{\sum_{k=1}^{n} \frac{1}{v_{k}+f_{k}^{\prime \prime}\left(q_{k}\right)}-G^{\prime}(p)}\right]>0, i, j \in\{1, \ldots, n\}, j \neq i .
$$

Proof cf., Kalashnikov et al. (2017) .

\section{Interior Equilibrium}

Now we are in a position to define the concept of interior equilibrium. To do that, we first describe the procedure of verification of the influence coefficients $v_{i}$ exactly as it was introduced in Bulavsky (1997). Assume that the system is in the exterior equilibrium $\left(p ; q_{1} \ldots, q_{n}\right)$ that occurs for some given $D$ and $v_{i}, i \in\{1, \ldots, n\}$. Now, one of the producers, say $k, 1 \leq k \leq n$, temporarily changes its behavior by abstaining from the maximization of the conjectured profit, subtracts its produce $q_{k}$ from the total demand and makes infinitesimal fluctuations around the latter. In mathematical terms, it is tantamount to restricting the model's producers to the subset $I_{-k}:=\{i \mid 1 \leq i \leq n, i \neq k\}$ with the output $q_{k}$ subtracted from the active demand with the balance equality restated in the form:

$$
\sum_{i \neq k} q_{i}=G(p)+D-q_{k} .
$$


Variations of the production output by producer $k$ is then equivalent to the active demand fluctuation in the form $d D_{k}:=d\left(D-q_{k}\right)=-d q_{k}$. If we treat these variations as infinitesimal, we can assume that by observing the corresponding variations of the equilibrium price in the equilibrium attained among the remaining participants, producer $k$ can evaluate the derivative of the equilibrium price with respect to the active demand, i.e., their influence coefficient.

Applying formula (10) from Theorem 3.3 to calculate the derivatives, one has to remember that producer $k$ is temporarily absent from the equilibrium model, hence one has to exclude the term with number $i=k$ from the sum. Having that in mind, we come to the following criterion.

Consistency criterion 4.1 In the exterior equilibrium $\left(p ; q_{1} \ldots, q_{n}\right)$, the influence coefficients $v_{i}, i \in\{1, \ldots, n\}$, are called consistent if the following equalities hold:

$$
v_{i}=\frac{1}{\sum_{\substack{j=1 \\ j \neq i}}^{n} \frac{1}{v_{j}+f_{j}^{\prime \prime}\left(q_{j}\right)}-G^{\prime}(p)}, i \in\{1, \ldots, n\} .
$$

A definition of the consistent (interior) equilibrium follows.

Definition 4.2 A collection $\left(p ; q_{1} \ldots, q_{n} ; v_{1}, \ldots, v_{n}\right)$ is referred to as interior equilibrium if, for the influence coefficients $v_{i} \geq 0, i \in\{1, \ldots, n\}$, the vector $\left(p ; q_{1} \ldots, q_{n}\right)$ is the exterior equilibrium, and the consistency criterion 4.1 is valid for all those $v_{i}$.

Theorem 4.3 Let the number of oligopoly producers be at least three, i.e., $n \geq 3$, under assumptions A2.1, A2.2 and A3.1, there exists an interior equilibrium. Moreover, if the number of producer is two, i.e., $n=2$, in addition to assumptions A2.1, $A 2.2$ and A3.1, suppose that exists $\varepsilon>0$ such that $G^{\prime}(p) \leq-\varepsilon$ for all $p>0$, then, there exists interior equilibrium.

Proof cf., Appendix 1.

Without additional assumptions or simplifications of the model, uniqueness of the interior equilibrium is not guaranteed.

In our future research, we are going to extend the obtained results to the case of not necessarily differentiable demand functions. However, some of the essential techniques can be developed now, in the differentiable case but under a bit stronger assumptions about the structure of the producers' cost functions. Namely, let us introduce the following assumption instead of A2.2. Moreover, this new assumption is used in our extension of the existence theorem 4.3 to the case of duopoly, i.e., when $n=2$. 
A4.4 For every $i \in\{1, \ldots, n\}$, the cost function $f_{i}$ is quadratic (and strictly convex) with $f_{i}(0)=0, f_{i}^{\prime}(0)>0$ and $f_{i}^{\prime \prime}>0$, i.e.,

$$
f_{i}\left(q_{i}\right)=\frac{1}{2} a_{i} q_{i}^{2}+b_{i} q_{i}
$$

where $a_{i}>0, b_{i}>0, i \in\{1, \ldots, n\}$.

Theorem 4.5 Let $n=2$ (duopoly), and assumptions A2.1, A3.1 and A4.3 hold true. If in addition there exists $\varepsilon>0$ such that $G^{\prime}(p) \leq-\varepsilon$ for all $p>0$, then, there exits the unique interior equilibrium.

Proof cf., Kalashnikov et al. (2017).

Now denote the value of the demand function's derivative by $\tau=G^{\prime}(p)$ and rewrite the consistency equations (15) in the form:

$$
v_{i}=\frac{1}{\sum_{\substack{j=1 \\ j \neq i}}^{n} \frac{1}{v_{j}+f_{j}^{\prime \prime}\left(q_{j}\right)}-\tau}, i \in\{1, \ldots, n\},
$$

where $\tau \in(-\infty, 0]$. When $\tau \rightarrow-\infty$ the system (17) converges to the solution $v_{i}=0, i \in\{1, \ldots, n\}$. For all finite values of $\tau$ we can establish the following proposition.

Theorem 4.6 Let assumptions A2.1, A3.1 and A4.3 be valid. Then, for any $\tau \in$ $(-\infty, 0]$ there exists a unique solution $v_{i}=v_{i}(\tau), i \in\{1, \ldots, n\}$, of equations (17), continuously depending upon $\tau$. Furthermore, $v_{i} \rightarrow 0$ when $\tau \rightarrow-\infty$, and $v_{i}(\tau)$ strictly increases and tends to $v_{i}(0)$ as $\tau \rightarrow 0, i \in\{1, \ldots, n\}$.

Proof The proof is easily deduced from that of Kalashnikov et al. (2011)[Theorem 3].

\section{Consistent Conjectures as Optimal Nash Strategies in the Meta-Model}

This section establishes the three most important results of this paper. Indeed, under certain rather mild conditions, we prove the equivalence of the consistent conjectural variations equilibrium (CVE) in an original oligopoly model to the classical CournotNash equilibrium in the meta-model. The latter comprises the same agents of the original oligopoly but with their conjectures (about the possible price variations) as their strategies.

These results seem to be very interesting in two aspects. First, they could be considered as a good justification of the CVE concept as being tightly related to the 
classical Nash equilibrium. Second, this equivalence occurring in the oligopoly can help one develop a concept similar to the CVE but in application to other kinds of economic and financial models that lack some attributes of the oligopoly and thus do not allow one to introduce the (consistent) CVE directly. In other words, one could define the (consistent) CVE in such a model via the Nash equilibrium in the corresponding meta-model.

To begin with, Theorem 3.3 allows us to define the following many-person game $\Gamma=(N, V, \Pi, D)$, which will be referred to as the meta-model. Here, $D \geq 0$ is the fixed value of the active demand, $N=\{1, \ldots, n\}$ is the set of the same producers (the payers) as in the above described model, $V=\mathbb{R}_{+}^{n}$ represents the set of possible strategies, i.e., the vectors of conjectures $v=\left(v_{1}, \ldots, v_{n}\right)$ accepted by the producers. Finally, $\Pi=\Pi(\nu)=\left(\pi_{1} \ldots \pi_{n}\right)$ is the collection of payoff values defined (uniquely, according to Theorem 3.3) by the strategy vector $v$. Indeed, the payoff values $\pi_{i}=\pi_{i}(v), i \in\{1, \ldots, n\}$, are defined by formula (5), were the equilibrium outputs $q_{i} \geq 0, i \in\{1, \ldots, n\}$, as well as the equilibrium price $p$, are the elements of the exterior equilibrium whose existence and uniqueness is guaranteed by Theorem 3.3 from Section 3.

Now the main results of this paper are as follows. As was mentioned in the introduction, the Cournot-Nash conjectures $\omega_{i}=1$ are usually inconsistent (in sense of criterion 4.1) in our single commodity market model. In other words, the CournotNash conjectures $v_{i}=-1 / G^{\prime}(p)$ in general do not satisfy the consistency system (15). However, in the meta-model introduced above, the consistent conjectures $v_{i}$, $i \in\{1, \ldots, n\}$, determined by Eq. 15 provide the Nash equilibrium. This curious fact could be considered as an extra argument supporting the concept of interior equilibrium introduced in Section 4.

Theorem 5.1 Suppose that assumptions A2.1, A2.2, and A3.1 hold. Then, any Nash equilibrium in the meta-model $\Gamma=(N, V, \Pi, D)$ generates interior equilibrium in the original oligopoly.

Proof cf., Kalashnikov et al. (2017).

Since the meta-model strategies set $V=\mathbb{R}_{+}^{n}$ is unbounded, the existence of at least one Nash equilibrium state in this game is by no means easy to check. The following three results (under some extra assumptions) guarantee that the existence of interior equilibrium in the original oligopoly implies the existence of Nash equilibrium in the meta-model.

Theorem 5.2 Suppose that the stronger assumption A4.3 is true, together with A2.1 and A3.1, and suppose that the function $G$ is concave. Then, the consistency criterion for the original oligopoly is a necessary and sufficient condition for the collection of influence conjectures $v=\left(v_{1}, \ldots, v_{n}\right)$ to produce Nash equilibrium in the metamodel. 
Corollary 5.3 In addition to assumptions A2.1, A3.1, and A4.3, if the demand function is affine, that is,

$$
G(p):=-K p+T,
$$

where $K>0$ and $T>0$, then, the consistency criterion for the original oligopoly is a necessary and sufficient condition for the collection of influence conjectures $v=\left(v_{1}, \ldots, v_{n}\right)$ to form Nash equilibrium in the meta-model.

Since the concavity of the demand function may be a much too restrictive requirement, the next theorem relaxes it by replacing it with the Lipschitz continuity of the derivative $G^{\prime}(p)$.

Theorem 5.4 Suppose that apart from assumptions A2.1, A3.1 and A4.3, the regular demand function's derivative is Lipschitz continuous. In more detail, for $n \geq 3$ assume that for any $p_{1}>0$ and $p_{2}>0$ the following inequality holds:

$$
\left|G^{\prime}\left(p_{1}\right)-G^{\prime}\left(p_{2}\right)\right| \leq \frac{1}{2 s^{2} G\left(p_{0}\right)}\left|p_{1}-p_{2}\right|,
$$

where $s=\max \left\{a_{1}, \ldots, a_{n}\right\}$, and the price $p_{0}$ is the one defined in the assumption A3.1. Next, if $n=2$ (duopoly), we again suppose that there exists $\varepsilon>0$ such that $G^{\prime}(p) \leq-\varepsilon$ for all $p>0$, and the Lipschitz continuity of the demand function is described in the form:

$$
\left|G^{\prime}\left(p_{1}\right)-G^{\prime}\left(p_{2}\right)\right| \leq \frac{2}{\left(\frac{a_{1}+a_{2}}{\varepsilon \min \left\{a_{1}, a_{2}\right\}}+3 \max \left\{a_{1}, a_{2}\right\}\right)^{2} G\left(p_{0}\right)}\left|p_{1}-p_{2}\right|, \forall p_{1}, p_{2}>0 .
$$

Then, the consistency criterion for the original oligopoly is a necessary and sufficient condition for the collection of influence conjectures $v=\left(v_{1} \ldots v_{n}\right)$ to be the Nash equilibrium in the meta-model.

Proof See Appendix 3.

\section{Numerical Experiments}

Now we illustrate our main results from Section 5. For the numerical experiments, we consider the inverse demand and costs function from an electricity market presented in Liu et al. (2007).

The inverse demand function is given by:

$$
p(G, D)=50-0.02(G+D),
$$

thus, the demand function has the form:

$$
G(p)+D=-50 p+2500 .
$$

There are $n=6$ firms with quadratic costs functions, i.e., $f_{i}\left(q_{i}\right)=\frac{1}{2} a_{i} q_{i}{ }^{2}+b_{i} q_{i}$, $i \in\{1, \ldots, n\}$, where the coefficients $a_{i}$ and $b_{i}$ are given in Table 1 . 
Table 1 Quadratic costs function's coefficients

\begin{tabular}{lll}
\hline$i$ & $a_{i}$ & $b_{i}$ \\
\hline 1 & 0.02 & 2.0 \\
2 & 0.0175 & 1.75 \\
3 & 0.025 & 3.0 \\
4 & 0.025 & 3.0 \\
5 & 0.0625 & 1.0 \\
6 & 0.00834 & 3.25 \\
\hline
\end{tabular}

In addition, as a kind of dual concept developed in our previous papers, we are going to consider the influence of the producers over the total output $G$ ( $c f$., Isac et al. (2002)), i.e, the influence coefficients $\omega_{i}:=\frac{\partial G}{\partial q_{i}}, i \in\{1, \ldots, n\}$.

In this sense, the conjectures $\omega_{i}=1, \forall i \in\{1, \ldots, n\}$, correspond to the CournotNash conjectures, while the zero-conjectures $\omega_{i}=0, \forall i \in\{1, \ldots, n\}$, lead to the perfect competition model.

Moreover, by the chain rule, one can easily verify the relationship

$$
\omega_{i}=-G^{\prime}(p) \nu_{i}, \forall i \in\{1, \ldots, n\} .
$$

Experiment 1 For the electricity market described above, the producers' influence coefficients $\omega_{i}$, supplies $q_{i}$ and profits $\pi_{i}, i \in\{1, \ldots, n\}$, along with the market's price $p$ and demand $G$, of the interior equilibrium results are shown in Table 2 .

Next, we vary the influence coefficient of one of the producers and compute the corresponding exterior equilibrium to see how their profits change.

In Tables 3, 4, 5, 6, 7, 8, we see that whenever one of the producers (unilaterally) increases or decreases its consistent influence coefficient, its profit drops. This, as proved in Section 5, is due to the fact that the consistent influence coefficients describing the interior equilibrium form the Nash equilibrium in the meta-model.

Experiment 2 Here, we are going to find the interior equilibrium for an electricity market (as the base model) that does not meet the conditions of Theorems 5.2 and 5.4, and test if it serves as the Nash equilibrium in the meta-model.

Now, we consider the following demand function:

$$
G(p)+D=2400 p^{-1.2}+1600,
$$

along with the cost functions described above.

Table 2 Interior equilibrium for the electricity market

\begin{tabular}{lllllll}
\hline$i$ & 1 & 2 & 3 & 4 & 5 & 6 \\
\hline$\omega_{i}$ & 0.19275 & 0.19635 & 0.18759 & 0.18759 & 0.17472 & 0.22391 \\
$q_{i}$ & 353.40 & 405.12 & 258.44 & 258.44 & 142.90 & 560.18 \\
$\pi_{i}$ & 1730.4 & 2080.6 & 1085.4 & 1085.4 & 709.48 & 2713.8 \\
$p$ & \multicolumn{7}{c}{10.431} & \\
$G$ & \multicolumn{7}{c}{1978.5} \\
\hline
\end{tabular}


Table 3 Net profits when agent 1 (unilaterally) changes its consistent conjecture (the new data is in bold)

\begin{tabular}{lllllll}
\hline$i$ & 1 & 2 & 3 & 4 & 5 & 6 \\
\hline$\omega_{i}$ & $\begin{array}{l}0.19275 \\
\mathbf{0 . 3 9 1 6 5}\end{array}$ & 0.19635 & 0.18759 & 0.18759 & 0.17472 & 0.22391 \\
& 1730.4 & 2163.4 & 1136.0 & 1136.0 & 735.45 & 2844.6 \\
$\pi_{i}$ & $\mathbf{1 7 0 3 . 2}$ & & & & & \\
\hline
\end{tabular}

Table 4 Net profits when producer 2 (unilaterally) changes its consistent conjecture (the new data is in bold)

\begin{tabular}{lllllll}
\hline$i$ & 1 & 2 & 3 & 4 & 5 & 6 \\
\hline$\omega_{i}$ & 0.19275 & $\begin{array}{l}0.19635 \\
\mathbf{0 . 1 2 0 0 0}\end{array}$ & 0.18759 & 0.18759 & 0.17472 & 0.22391 \\
& & & & & \\
$\pi_{i}$ & 1688.8 & 2080.6 & 1055.9 & 1055.9 & 694.22 & 2637.3 \\
& & $\mathbf{2 0 7 2 . 0}$ & & & & \\
\hline
\end{tabular}

Table 5 Net profits when agent 3 (unilaterally) changes its consistent conjecture (the new data is in bold)

\begin{tabular}{|c|c|c|c|c|c|c|}
\hline$i$ & 1 & 2 & 3 & 4 & 5 & 6 \\
\hline \multirow[t]{2}{*}{$\omega_{i}$} & 0.19275 & 0.19635 & 0.18759 & 0.18759 & 0.17472 & 0.22391 \\
\hline & & & 0.43615 & & & \\
\hline \multirow[t]{2}{*}{$\pi_{i}$} & 1783.6 & 2142.7 & 1085.4 & 1123.3 & 728.97 & 2811.9 \\
\hline & & & 1066.3 & & & \\
\hline
\end{tabular}

Table 6 Net profits when producer 4 (unilaterally) changes its consistent conjecture (the new data is in bold)

\begin{tabular}{|c|c|c|c|c|c|c|}
\hline$i$ & 1 & 2 & 3 & 4 & 5 & 6 \\
\hline \multirow[t]{2}{*}{$\omega_{i}$} & 0.19275 & 0.19635 & 0.18759 & 0.18759 & 0.17472 & 0.22391 \\
\hline & & & & 0.061570 & & \\
\hline \multirow[t]{2}{*}{$\pi_{i}$} & 1697.1 & 2041.7 & 1061.8 & 1085.4 & 697.27 & 2652.6 \\
\hline & & & & 1077.8 & & \\
\hline
\end{tabular}


Table 7 Net profits when agent 5 (unilaterally) changes its consistent conjecture (the new data is in bold)

\begin{tabular}{lllllll}
\hline$i$ & 1 & 2 & 3 & 4 & 5 & 6 \\
\hline$\omega_{i}$ & 0.19275 & 0.19635 & 0.18759 & 0.18759 & 0.17472 & 0.22391 \\
& & & & & $\mathbf{0 . 1 4 8 7 0}$ & \\
$\pi_{i}$ & 1728.9 & 2078.8 & 1084.3 & 1084.3 & 709.48 & 2711.0 \\
\hline
\end{tabular}

Table 8 Net profits when producer 6 (unilaterally) changes its consistent conjecture (the new data is in bold)

\begin{tabular}{lllllll}
\hline$i$ & 1 & 2 & 3 & 4 & 5 & 6 \\
\hline$\omega_{i}$ & 0.19275 & 0.19635 & 0.18759 & 0.18759 & 0.17472 & 0.22391 \\
& & & & & $\mathbf{0 . 4 7 3 0 5}$ \\
$\pi_{i}$ & 1968.4 & 2358.2 & 1255.6 & 1255.6 & 796.41 & 2713.8 \\
& & & & & $\mathbf{2 5 7 8 . 1}$ \\
\hline
\end{tabular}

Table 9 Interior equilibrium for the electricity market with the nonlinear demand function defined by (24)

\begin{tabular}{lllllll}
\hline$i$ & 1 & 2 & 3 & 4 & 5 & 6 \\
\hline$\omega_{i}$ & 0.086234 & 0.088004 & 0.083674 & 0.083674 & 0.077221 & 0.10112 \\
$q_{i}$ & 317.77 & 363.63 & 231.17 & 231.17 & 132.16 & 479.30 \\
$\pi_{i}$ & 1467.8 & 1769.1 & 903.22 & 903.22 & 616.76 & 2179.9 \\
$p$ & & & \multicolumn{2}{c}{9.7968} & \\
$G$ & & \multicolumn{3}{c}{1755.2} & \\
\hline
\end{tabular}

Table 10 Net profits when the demand function is nonlinear and the producers vary (unilaterally) their conjectures (the consistent conjectures and the corresponding profits are in bold)

\begin{tabular}{lllllll}
\hline$i$ & 1 & 2 & 3 & 4 & 5 & 6 \\
\hline$\omega_{i}$ & 0.060438 & 0.076765 & 0.035183 & 0.079306 & 0.074729 & 0.093522 \\
& $\mathbf{0 . 0 8 6 2 3 4}$ & $\mathbf{0 . 0 8 8 0 0 4}$ & $\mathbf{0 . 0 8 3 6 7 4}$ & $\mathbf{0 . 0 8 3 6 7 4}$ & $\mathbf{0 . 0 7 7 2 2 1}$ & $\mathbf{0 . 1 0 1 1 2}$ \\
& 0.11277 & 0.10719 & 0.095928 & 0.12058 & 0.079999 & 0.1033 \\
$\pi_{i}$ & 1464.0 & 1768.1 & 897.04 & 903.18 & 616.76 & 2178.5 \\
& $\mathbf{1 4 6 7 . 8}$ & $\mathbf{1 7 6 9 . 1}$ & $\mathbf{9 0 3 . 2 2}$ & $\mathbf{9 0 3 . 2 2}$ & $\mathbf{6 1 6 . 7 6}$ & $\mathbf{2 1 7 9 . 9}$ \\
& 1464.3 & 1766.4 & 902.88 & 900.35 & 616.76 & 2179.8 \\
\hline
\end{tabular}


The function (24) is not concave nor Lipschitz, but we can still compute the corresponding interior equilibrium. The corresponding data is shown in Table 9.

Finally, we vary the consistent influence coefficient of one of the producers and compute the corresponding exterior equilibrium to see how their profits change. These results are shown in Table 10.

From Table 10, one can see that each time as one of the agents changes (unilaterally) its consistent influence coefficient, its profit decreases. (Each column $i \in\{1, \ldots, 6\}$ shows the profit of producer $i$ for the respective influence coefficient while the other producers keep being stuck to their consistent conjectures.) Thus, the results of Theorems 5.2 and 5.4 can hold true for a wider set of functions than the ones satisfying the theorems' requirements.

\section{Conclusions}

This paper logically completes the authors' previous papers Kalashnikov et al. (2011, 2017) by providing in Section 5 three results establishing (under certain rather mild conditions) the equivalence of the consistent conjectural variations equilibrium (CVE) in an original oligopoly model to the classical Cournot-Nash equilibrium in the meta-model. The latter comprises the same agents of the original oligopoly but with their conjectures (about the possible price variations) as their strategies.

These results seem to be very interesting in two aspects. First, they could be considered as a good justification of the CVE concept as being tightly related to the classical Nash equilibrium. Second, this equivalence occurring in the oligopoly can help one develop a concept similar to the CVE but in application to other kinds of economic and financial models that lack some attributes of the oligopoly and thus do not allow one to introduce the (consistent) CVE directly. In other words, one could define the (consistent) CVE in such a model via the Nash equilibrium in the corresponding meta-model.

In our future research, we plan to implement the above-mentioned ideas, as well as extend the developed constructions to the cases of mixed oligopolies, where at least one agent endeavors to maximize not its net profit but some other function related to the social surplus. Another important extension could be related to more general economic models with not necessarily differentiable (even discontinuous) inverse demand and/or cost functions.

Acknowledgments This research was financially supported by the Mexico SEP-CONACYT grants CB2013-01-221676 and FC-2016-01-1938.

The authors would also like to express their profound gratitude to the three anonymous referees whose valuable comments and recommendations have helped us a lot to improve the paper's structure and presentation.

Open Access This article is distributed under the terms of the Creative Commons Attribution 4.0 International License (http://creativecommons.org/licenses/by/4.0/), which permits unrestricted use, distribution, and reproduction in any medium, provided you give appropriate credit to the original author(s) and the source, provide a link to the Creative Commons license, and indicate if changes were made. 


\section{Appendix 1: Proof of Theorem 4.3}

Theorem 4.3 Let the number of oligopoly producers be at least three, i.e., $n \geq 3$, under assumptions A2.1, A2.2 and A3.1, there exists an interior equilibrium. Moreover, if the number of producer is two, i.e., $n=2$, in addition to assumptions A2.1, A2.2 and A3.1, suppose that exists $\varepsilon>0$ such that $G^{\prime}(p) \leq-\varepsilon$ for all $p>0$, then, there exists interior equilibrium.

Proof For any given set of influence coefficients $v=\left(v_{1} \ldots v_{n}\right) \geq 0$, by Theorem 3.3, there exists the unique exterior equilibrium $\left(p(v), q_{1}(v), \ldots, q_{n}(v)\right)$.

Now, we define the following functions:

$$
F_{i}(v)=\frac{1}{\sum_{\substack{j=1 \\ j \neq i}}^{n} \frac{1}{v_{j}+f_{j}^{\prime \prime}\left(q_{j}(v)\right)}-G^{\prime}(p(v))}, i \in\{1, \ldots, n\} .
$$

These functions are well-defined and continuous with respect to $v=\left(v_{1} \ldots v_{n}\right) \geq 0$, due to assumptions A2.1 and A2.2.

Therefore, the function $H=\left(F_{1} \ldots, F_{n}\right): \mathbb{R}_{+}^{n} \rightarrow \mathbb{R}_{+}^{n}$ is also continuous. 0 .

Next, we define the value $s=\max \left\{f_{i}^{\prime \prime}\left(q_{i}\right) \mid 0 \leq q_{i} \leq G\left(p_{0}\right), i \in\{1, \ldots, n\}\right\}>$ For $n \geq 3$, if $0 \leq v_{i} \leq \frac{s}{n-2}$ for all $i \in\{1, \ldots, n\}$, we have:

$$
\begin{aligned}
0 \leq F_{i}(v) & =\frac{1}{\sum_{\substack{j=1 \\
j \neq i}}^{n} \frac{1}{v_{j}+f_{j}^{\prime \prime}\left(q_{j}(v)\right)}-G^{\prime}(p(v))} \leq \frac{1}{\sum_{\substack{j=1 \\
j \neq i}}^{n} \frac{1}{v_{j}+f_{j}^{\prime \prime}\left(q_{j}(v)\right)}} \\
& \leq \frac{1}{\sum_{\substack{j=1 \\
j \neq i}}^{n} \frac{1}{n-2}+s}=\frac{1}{\frac{s}{n-2}, i \in\{1, \ldots, n\} .} \frac{\frac{s}{n-2}+s}{n-2}
\end{aligned}
$$

Thus, the function $H=\left(F_{1} \ldots, F_{n}\right)$ maps the convex compact subset $\left[0, \frac{s}{n-2}\right]^{n}$ onto itself. Therefore, by Brouwer's fixed-point theorem, $H$ has a fixed point, i.e., there exists $v^{*}=\left(v_{1}^{*} \ldots v_{n}^{*}\right) \geq 0$ such that $F_{i}\left(v^{*}\right)=v_{i}^{*}$ for all $i \in\{1, \ldots, n\}$.

On the other hand, for $n=2$ and $G^{\prime}(p) \leq-\varepsilon$ for some $\varepsilon>0$, if $0 \leq v_{i} \leq \frac{1}{\varepsilon}$ for all $i \in\{1, \ldots, n\}$, we have:

$$
\begin{aligned}
& 0 \leq F_{1}(v)=\frac{1}{\frac{1}{v_{2}+f_{2}^{\prime \prime}\left(q_{2}(v)\right)}-G^{\prime}(p(v))} \leq \frac{1}{-G^{\prime}(p(v))} \leq \frac{1}{\varepsilon}, \\
& 0 \leq F_{2}(v)=\frac{1}{\frac{1}{v_{1}+f_{1}^{\prime \prime}\left(q_{1}(v)\right)}-G^{\prime}(p(v))} \leq \frac{1}{-G^{\prime}(p(v))} \leq \frac{1}{\varepsilon} .
\end{aligned}
$$


Thus, the function $H=\left(F_{1}, F_{2}\right)$ maps the convex compact subset $\left[0, \frac{1}{\varepsilon}\right]^{2}$ onto itself, then, again by Brouwer's fixed-point theorem, $H$ has a fixed point $F_{i}\left(v^{*}\right)=$ $v_{i}^{*}, i \in\{1,2\}$.

By the definition of the functions (25), the influence coefficients $v^{*}=$ $\left(v_{1}^{*} \ldots v_{n}^{*}\right) \geq 0$, given by Brouwer'sfixed-point theorem, satisfy the Consistency Criterion 4.1 and, therefore, the vector $\left(p\left(v^{*}\right), q_{1}\left(v^{*}\right), \ldots, q_{n}\left(v^{*}\right), v_{1}^{*}, \ldots, v_{n}^{*}\right)$ is the interior equilibrium. The proof is complete.

\section{Appendix 2: Proof of Theorem 5.2}

Theorem 5.2 Suppose that the stronger assumption A4.3 is true, together with A2.1 and A3.1, and suppose that the function $G$ is concave. Then, the consistency criterion for the original oligopoly is a necessary and sufficient condition for the collection of influence conjectures $v=\left(v_{1}, \ldots, v_{n}\right)$ to produce Nash equilibrium in the meta-model.

Proof Note that the necessity is a particular case of Theorem 5.1, thus, to prove Theorem 5.2, we just need to establish the sufficiency.

We assume A4.3, that is, for all $i$, the cost functions $f_{i}$ are quadratic (and strictly convex) with $f_{i}(0)=0, f_{i}^{\prime}(0)>0$, and $f_{i}^{\prime \prime}(0)>0$, i.e.,

$$
f_{i}\left(q_{i}\right)=\frac{1}{2} a_{i} q_{i}^{2}+b_{i} q_{i}
$$

where $a_{i}>0, b_{i}>0, i \in\{1, \ldots, n\}$. Now we are in a position to demonstrate that in this specific case, each interior equilibrium $\left(p^{*} ; q_{1}^{*}, \ldots, q_{n}^{*} ; v_{1}^{*}, \ldots, v_{n}^{*}\right)$ of the original oligopoly provides the Nash equilibrium in the meta-model $\Gamma=(N, V, \Pi, D)$. Namely, the consistent conjectures (influence coefficients) $v^{*}=\left(v_{1}^{*}, \ldots, v_{n}^{*}\right)$ satisfying (15) form the Nash equilibrium in the meta-model.

Indeed, first of all, equations (15) in this particular case are reduced to the system

$$
v_{i}^{*}=\frac{1}{\sum_{\substack{k=1 \\ k \neq i}}^{n} \frac{1}{v_{k}^{*}+a_{k}}-G^{\prime}\left(p^{*}\right)}, i \in\{1, \ldots, n\},
$$

which clearly implies that all components of the vector $v^{*}$ are positive: $v_{i}^{*}>0$, $i \in\{1, \ldots, n\}$.

Next, equations

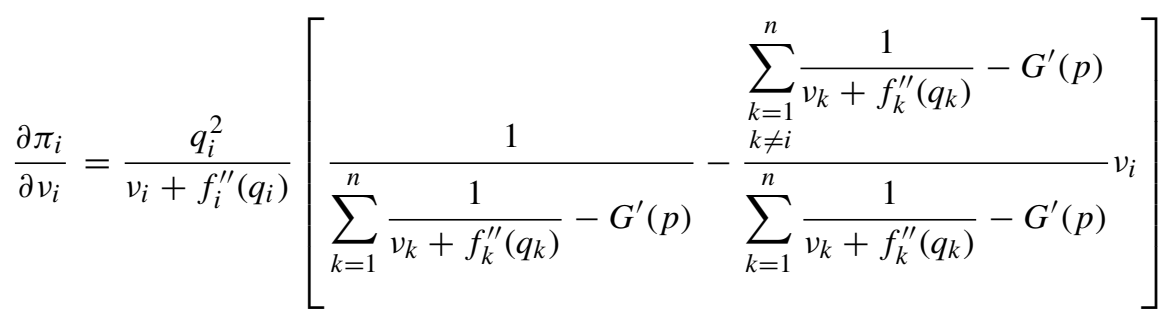




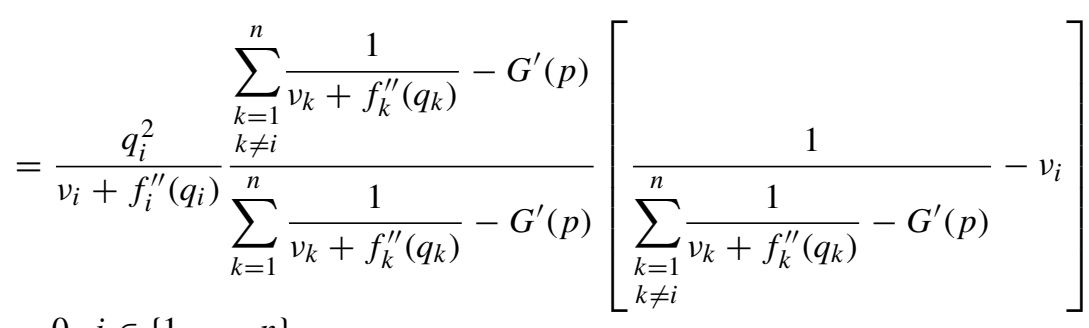

$$
\begin{aligned}
& =0, i \in\{1, \ldots, n\} \text {. }
\end{aligned}
$$

guarantee that the first-order optimality conditions for the meta-game payoff functions hold:

$$
\frac{\partial \pi_{i}}{\partial v_{i}}\left(v^{*}\right)=0, i \in\{1, \ldots, n\}
$$

Therefore, the value $v_{i}^{*}$ may be the maximum point of the $i$-th producer's payoff function

$$
\tilde{\pi}_{i}\left(v_{i}\right) \equiv \pi\left(v_{i}, v_{-i}^{*}\right), i \in\{1, \ldots, n\},
$$

where $v_{-i}^{*}=\left(v_{1}^{*}, \ldots, v_{i-1}^{*}, v_{i+1}^{*}, \ldots, v_{n}^{*}\right)$. In order to establish the maximum point property, we are going to fix an arbitrary $i$ and to show that the function $\tilde{\pi}_{i}=\tilde{\pi}_{i}\left(v_{i}\right)$ :

(a) doesn't increase along the ray $\left(v_{i}^{*},+\infty\right)$,

(b) doesn't decrease in the interval $\left(0, v_{i}^{*}\right)$.

In order to prove (a), taking into account (29), it suffices to show that

$$
\frac{1}{\sum_{\substack{k=1 \\ k \neq i}}^{n} \frac{1}{v_{k}^{*}+a_{k}}-G^{\prime}\left(p\left(v_{i}^{*}+\delta, v_{-i}^{*}\right)\right)}-\left(v_{i}^{*}+\delta\right) \leq 0, \forall \delta>0 .
$$

By inverting both sides of the consistency equation (28) one gets

$$
\frac{1}{v_{i}^{*}}=\sum_{\substack{k=1 \\ k \neq i}}^{n} \frac{1}{v_{k}^{*}+a_{k}}-G^{\prime}\left(p^{*}\right)
$$

which clearly implies the relationships

$$
\sum_{\substack{k=1 \\ k \neq i}}^{n} \frac{1}{v_{k}^{*}+a_{k}}=\frac{1}{v_{i}^{*}}+G^{\prime}\left(p^{*}\right)=\frac{1+v_{i}^{*} G^{\prime}\left(p^{*}\right)}{v_{i}^{*}}>0 .
$$


Making use of Eq. 34, rewrite the left-hand side of Eq. 32 in the form

$$
\begin{aligned}
& \frac{1}{\sum_{\substack{k=1 \\
k \neq i}}^{n} \frac{1}{v_{k}^{*}+a_{k}}-G^{\prime}\left(p\left(v_{i}^{*}+\delta, v_{-i}^{*}\right)\right)}-\left(v_{i}^{*}+\delta\right)=\frac{1}{\frac{1}{v_{i}^{*}}+G^{\prime}\left(p^{*}\right)-G^{\prime}\left(p\left(v_{i}^{*}+\delta, v_{-i}^{*}\right)\right)}-\left(v_{i}^{*}+\delta\right) \\
& =\frac{v_{i}^{*}}{1+v_{i}^{*} G^{\prime}\left(p^{*}\right)-v_{i}^{*} G^{\prime}\left(p\left(v_{i}^{*}+\delta, v_{-i}^{*}\right)\right)}-\left(v_{i}^{*}+\delta\right) \\
& =\frac{\left(v_{i}^{*}\right)^{2}\left[G^{\prime}\left(p\left(v_{i}^{*}+\delta, v_{-i}^{*}\right)\right)-G^{\prime}\left(p^{*}\right)\right]-\delta+v_{i}^{*} \delta\left[G^{\prime}\left(p\left(v_{i}^{*}+\delta, v_{-i}^{*}\right)\right)-G^{\prime}\left(p^{*}\right)\right]}{1+v_{i}^{*} G^{\prime}\left(p^{*}\right)-v_{i}^{*} G^{\prime}\left(p\left(v_{i}^{*}+\delta, v_{-i}^{*}\right)\right)} \\
& =\frac{v_{i}^{*}\left[G^{\prime}\left(p\left(v_{i}^{*}+\delta, v_{-i}^{*}\right)\right)-G^{\prime}\left(p^{*}\right)\right]\left(v_{i}^{*}+\delta\right)-\delta}{1+v_{i}^{*} G^{\prime}\left(p^{*}\right)-v_{i}^{*} G^{\prime}\left(p\left(v_{i}^{*}+\delta, v_{-i}^{*}\right)\right)} .
\end{aligned}
$$

Since $1+v_{i}^{*} G^{\prime}\left(p^{*}\right)>0$ from Eq. 34 , and $-v_{i}^{*} G^{\prime}\left(p\left(v_{i}^{*}+\delta, v_{-i}^{*}\right)\right) \geq 0$ by assumption A2.1, then the denominator of Eq. 35 is strictly positive, thus the sign of ratio (35) is determined by that of its numerator. Now since the derivative $G^{\prime}(p)$ is non-increasing by hypothesis, and $\frac{\partial p}{\partial \nu_{i}}>0$ by Eq. 11, it isn't difficult to show that $\left[G^{\prime}\left(p\left(v_{i}^{*}+\delta, v_{-i}^{*}\right)\right)-G^{\prime}\left(p^{*}\right)\right] \leq 0$, hence the numerator of Eq. 35 is strictly negative for any $\delta>0$ :

$$
v_{i}^{*}\left[G^{\prime}\left(p\left(v_{i}^{*}+\delta, v_{-i}^{*}\right)\right)-G^{\prime}\left(p^{*}\right)\right]\left(v_{i}^{*}+\delta\right)-\delta<0, \forall \delta>0 .
$$

The latter brings about the desired inequality

$$
\frac{d \tilde{\pi}_{i}}{d v_{i}}\left(v_{i}, v_{-i}^{*}\right)<0, \forall v_{i}>v_{i}^{*},
$$

which finishes the proof of (a).

Now to demonstrate that (b) is also true, again taking into account (29), it is enough to check that

$$
\frac{1}{\sum_{\substack{k=1 \\ k \neq i}}^{n} \frac{1}{v_{k}^{*}+a_{k}}-G^{\prime}\left(p\left(v_{i}^{*}-\delta, v_{-i}^{*}\right)\right)}-\left(v_{i}^{*}-\delta\right) \geq 0, \forall \delta \text { such that } 0<\delta<v_{i}^{*} .
$$

Again employing Eq. 34 yields the following transformation of the left-hand side of Eq. 38:

$$
\begin{aligned}
& \frac{1}{\sum_{\substack{k=1 \\
k \neq i}}^{n} \frac{1}{v_{k}^{*}+a_{k}}-G^{\prime}\left(p\left(v_{i}^{*}-\delta, v_{-i}^{*}\right)\right)}-\left(v_{i}^{*}-\delta\right)=\frac{1}{\frac{1}{v_{i}^{*}}+G^{\prime}\left(p^{*}\right)-G^{\prime}\left(p\left(v_{i}^{*}-\delta, v_{-i}^{*}\right)\right)}-\left(v_{i}^{*}-\delta\right) \\
& \quad=\frac{v_{i}^{*}}{1+v_{i}^{*} G^{\prime}\left(p^{*}\right)-v_{i}^{*} G^{\prime}\left(p\left(v_{i}^{*}-\delta, v_{-i}^{*}\right)\right)}-\left(v_{i}^{*}-\delta\right) \\
& \quad=\frac{\left(v_{i}^{*}\right)^{2}\left[G^{\prime}\left(p\left(v_{i}^{*}-\delta, v_{-i}^{*}\right)\right)-G^{\prime}\left(p^{*}\right)\right]+\delta-v_{i}^{*} \delta\left[G^{\prime}\left(p\left(v_{i}^{*}-\delta, v_{-i}^{*}\right)\right)-G^{\prime}\left(p^{*}\right)\right]}{1+v_{i}^{*} G^{\prime}\left(p^{*}\right)-v_{i}^{*} G^{\prime}\left(p\left(v_{i}^{*}-\delta, v_{-i}^{*}\right)\right)} \\
& =\frac{v_{i}^{*}\left[G^{\prime}\left(p\left(v_{i}^{*}-\delta, v_{-i}^{*}\right)\right)-G^{\prime}\left(p^{*}\right)\right]\left(v_{i}^{*}-\delta\right)+\delta}{1+v_{i}^{*} G^{\prime}\left(p^{*}\right)-v_{i}^{*} G^{\prime}\left(p\left(v_{i}^{*}-\delta, v_{-i}^{*}\right)\right)} .
\end{aligned}
$$


Similar to the proof of case (a), the denominator of the fraction (39) is strictly positive, hence, the fraction's sign coincides with that of its numerator. Again, since the derivative $G^{\prime}(p)$ is non-increasing by hypothesis, and $\frac{\partial p}{\partial \nu_{i}}>0$ by Eq. 11 , it is evident that $\left[G^{\prime}\left(p\left(v_{i}^{*}-\delta, v_{-i}^{*}\right)\right)-G^{\prime}\left(p^{*}\right)\right] \geq 0$, hence, the numerator of Eq. 39 is strictly positive for any $0<\delta<v_{i}^{*}$ :

$$
v_{i}^{*}\left[G^{\prime}\left(p\left(v_{i}^{*}-\delta, v_{-i}^{*}\right)\right)-G^{\prime}\left(p^{*}\right)\right]\left(v_{i}^{*}-\delta\right)+\delta>0, \forall \delta \text { that } 0<\delta<v_{i}^{*},
$$

which deduces the desired inequality:

$$
\frac{d \tilde{\pi}_{i}}{d \nu_{i}}\left(v_{i}, v_{-i}^{*}\right)>0, \forall v_{i}<v_{i}^{*} .
$$

Therefore, the proof of (b) is also completed.

Now we can conclude that the Nash equilibrium condition has been established:

$$
\pi_{i}\left(v^{*}\right)=\max _{v_{i}>0} \pi_{i}\left(v_{i}, v_{-i}^{*}\right) \text {, for any } i \in\{1 \ldots, n\},
$$

which finishes the proof of Theorem 5.2

\section{Appendix 3: Proof of Theorem 5.4}

Theorem 5.4 Suppose that apart from assumptions A2.1, A3.1 and A4.3, the regular demand function's derivative is Lipschitz continuous. In more detail, for $n \geq 3$ assume that for any $p_{1}>0$ and $p_{2}>0$ the following inequality holds:

$$
\left|G^{\prime}\left(p_{1}\right)-G^{\prime}\left(p_{2}\right)\right| \leq \frac{1}{2 s^{2} G\left(p_{0}\right)}\left|p_{1}-p_{2}\right|,
$$

where $s=\max \left\{a_{1}, \ldots, a_{n}\right\}$, and the price $p_{0}$ is the one defined in the assumption A3.1. Next, if $n=2$ (duopoly), we again suppose that there exists $\varepsilon>0$ such that $G^{\prime}(p) \leq-\varepsilon$ for all $p>0$, and the Lipschitz continuity of the demand function is described in the form:

$$
\left|G^{\prime}\left(p_{1}\right)-G^{\prime}\left(p_{2}\right)\right| \leq \frac{2}{\left(\frac{a_{1}+a_{2}}{\varepsilon \min \left\{a_{1}, a_{2}\right\}}+3 \max \left\{a_{1}, a_{2}\right\}\right)^{2} G\left(p_{0}\right)}\left|p_{1}-p_{2}\right|, \forall p_{1}, p_{2}>0 .
$$

Then, the consistency criterion for the original oligopoly is a necessary and sufficient condition for the collection of influence conjectures $v=\left(v_{1}, \ldots, v_{n}\right)$ to form Nash equilibrium in the meta-model.

Proof Again, the necessity is just a particular case of Theorem 5.1, then, we proceed to show the sufficiency.

Just like in the proof of Theorem 5.2, we need to establish that the $i$-th producer's payoff function

$$
\tilde{\pi}_{i}\left(v_{i}\right) \equiv \pi\left(v_{i}, v_{-i}^{*}\right), i \in\{1, \ldots, n\},
$$

has a maximum point at $v_{i}=v_{i}^{*}$ for a fixed value of $i$, for which, again, it will suffice to show that: 
(a)

$$
v_{i}^{*}\left[G^{\prime}\left(p\left(v_{i}^{*}+\delta, v_{-i}^{*}\right)\right)-G^{\prime}\left(p^{*}\right)\right]\left(v_{i}^{*}+\delta\right)-\delta<0, \forall 0<\delta<s,
$$

(b)

$$
v_{i}^{*}\left[G^{\prime}\left(p\left(v_{i}^{*}-\delta, v_{-i}^{*}\right)\right)-G^{\prime}\left(p^{*}\right)\right]\left(v_{i}^{*}-\delta\right)+\delta>0, \forall 0<\delta<v_{i}^{*},
$$

where $s=\max \left\{a_{1}, \ldots, a_{n}\right\}>0$. From the proof of Theorem 4.3 (Bulavsky, 1997) we have:

$$
0 \leq v_{i}^{*} \leq \frac{s}{n-2}, i \in\{1, \ldots, n\} .
$$

Now, from assumption (19) and the fact that $\frac{\partial p}{\partial \nu_{i}}>0$, it follows that

$$
\begin{aligned}
v_{i}^{*}\left[G^{\prime}\left(p\left(v_{i}^{*}+\delta, v_{-i}^{*}\right)\right)-G^{\prime}\left(p^{*}\right)\right]\left(v_{i}^{*}+\delta\right)-\delta & \leq v_{i}^{*}\left|G^{\prime}\left(p\left(v_{i}^{*}+\delta, v_{-i}^{*}\right)\right)-G^{\prime}\left(p^{*}\right)\right|\left(v_{i}^{*}+\delta\right)-\delta \\
& \leq v_{i}^{*}\left(v_{i}^{*}+\delta\right) \frac{1}{2 s^{2} G\left(p_{0}\right)}\left|p\left(v_{i}^{*}+\delta, v_{-i}^{*}\right)-p^{*}\right|-\delta \\
& \leq v_{i}^{*}\left(v_{i}^{*}+\delta\right) \frac{1}{2 s^{2} G\left(p_{0}\right)}\left(p\left(v_{i}^{*}+\delta, v_{-i}^{*}\right)-p^{*}\right)-\delta .
\end{aligned}
$$

By the mean value theorem here exists a value $\hat{v}_{i}$ such that $v_{i}<\hat{v}_{i}<v_{i}+\delta$ and

$$
p\left(v_{i}^{*}+\delta, v_{-i}^{*}\right)-p^{*}=\delta \frac{\partial p}{\partial v_{i}}\left(\hat{v}_{i}, v_{-i}^{*}\right) .
$$

Using Eq. 11 we get

$$
\begin{aligned}
\frac{\partial p}{\partial v_{i}}\left(\hat{v}_{i}, v_{-i}^{*}\right) & =\frac{\frac{q_{i}\left(p\left(\hat{v}_{i}, v_{-i}^{*}\right),\left(\hat{v}_{i}, v_{-i}^{*}\right)\right)}{\hat{v}_{i}+a_{i}}}{\sum_{\substack{k=1 \\
k \neq i}}^{n} \frac{1}{v_{k}^{*}+a_{k}}+\frac{1}{\hat{v}_{i}+a_{i}}-G^{\prime}\left(p\left(\hat{v}_{i}, v_{-i}^{*}\right)\right)} \leq \frac{\frac{q_{i}\left(p\left(\hat{v}_{i}, v_{-i}^{*}\right),\left(\hat{v}_{i}, v_{-i}^{*}\right)\right)}{\hat{v}_{i}+a_{i}}}{\sum_{\substack{k=1 \\
k \neq i}}^{n} \frac{1}{v_{k}^{*}+a_{k}}+\frac{1}{\hat{v}_{i}+a_{i}}} \\
& \leq \frac{\frac{q_{i}\left(p\left(\hat{v}_{i}, v_{-i}^{*}\right),\left(\hat{v}_{i}, v_{-i}^{*}\right)\right)}{\hat{v}_{i}+a_{i}}}{\frac{1}{\hat{v}_{i}+a_{i}}}=q_{i}\left(p\left(\hat{v}_{i}, v_{-i}^{*}\right),\left(\hat{v}_{i}, v_{-i}^{*}\right)\right) \leq G\left(p_{0}\right) .
\end{aligned}
$$

Applying Eqs. 47 and 48 to Eq. 46 we find:

$$
v_{i}^{*}\left[G^{\prime}\left(p\left(v_{i}^{*}+\delta, v_{-i}^{*}\right)\right)-G^{\prime}\left(p^{*}\right)\right]\left(v_{i}^{*}+\delta\right)-\delta \leq v_{i}^{*}\left(v_{i}^{*}+\delta\right) \frac{1}{2 s^{2}} \delta-\delta=\left[v_{i}^{*}\left(v_{i}^{*}+\delta\right) \frac{1}{2 s^{2}}-1\right]_{(49)},
$$

moreover, since $0<v_{i}^{*} \leq s$ and $0<\delta<s$, it follows that

$$
v_{i}^{*}\left[G^{\prime}\left(p\left(v_{i}^{*}+\delta, v_{-i}^{*}\right)\right)-G^{\prime}\left(p^{*}\right)\right]\left(v_{i}^{*}+\delta\right)-\delta \leq\left[v_{i}^{*}\left(v_{i}^{*}+\delta\right) \frac{1}{2 s^{2}}-1\right] \delta<0,
$$

which proves (a).

Analogous to the previous case, we can find that

$$
v_{i}^{*}\left[G^{\prime}\left(p^{*}\right)-G^{\prime}\left(p\left(v_{i}^{*}-\delta, v_{-i}^{*}\right)\right)\right]\left(v_{i}^{*}-\delta\right)-\delta \leq\left[v_{i}^{*}\left(v_{i}^{*}-\delta\right) \frac{1}{2 s^{2}}-1\right] \delta,
$$


and, since $0<\delta<v_{i}^{*} \leq s$, we have

$$
v_{i}^{*}\left[G^{\prime}\left(p^{*}\right)-G^{\prime}\left(p\left(v_{i}^{*}-\delta, v_{-i}^{*}\right)\right)\right]\left(v_{i}^{*}-\delta\right)-\delta \leq\left[v_{i}^{*}\left(v_{i}^{*}-\delta\right) \frac{1}{2 s^{2}}-1\right] \delta<0,
$$

then

$$
v_{i}^{*}\left[G^{\prime}\left(p\left(v_{i}^{*}-\delta, v_{-i}^{*}\right)-G^{\prime}\left(p^{*}\right)\right)\right]\left(v_{i}^{*}-\delta\right)+\delta>0 .
$$

Therefore, the vector $v^{*}$ is Nash equilibrium for $n \geq 3$.

Finally, let $n=2$. We can repeat the steps for the case $n \geq 3$ to get the following inequality:

$$
\begin{aligned}
& v_{i}^{*}\left[G^{\prime}\left(p\left(v_{i}^{*}+\delta, v_{-i}^{*}\right)\right)-G^{\prime}\left(p^{*}\right)\right]\left(v_{i}^{*}+\delta\right)-\delta \\
& \leq\left[v_{i}^{*}\left(v_{i}^{*}+\delta\right) \frac{2}{\left(\frac{a_{1}+a_{2}}{\varepsilon \min \left\{a_{1}, a_{2}\right\}}+3 \max \left\{a_{1}, a_{2}\right\}\right)^{2}}-1\right] \delta .
\end{aligned}
$$

From

$$
\begin{aligned}
v_{i} & \leq \frac{1}{2}\left(\frac{a_{1}+a_{2}}{\varepsilon \min \left\{a_{1}, a_{2}\right\}}+\max \left\{a_{1}, a_{2}\right\}\right)+\max \left\{a_{1}, a_{2}\right\}= \\
& =\frac{1}{2}\left(\frac{a_{1}+a_{2}}{\varepsilon \min \left\{a_{1}, a_{2}\right\}}+3 \max \left\{a_{1}, a_{2}\right\}\right), i \in\{1,2\} .
\end{aligned}
$$

we have

$$
0<v_{i}^{*} \leq \frac{1}{2}\left(\frac{a_{1}+a_{2}}{\varepsilon \min \left\{a_{1}, a_{2}\right\}}+3 \max \left\{a_{1}, a_{2}\right\}\right)
$$

and

$$
0<\delta<\max \left\{a_{1}, a_{2}\right\}<\frac{1}{2}\left(\frac{a_{1}+a_{2}}{\varepsilon \min \left\{a_{1}, a_{2}\right\}}+3 \max \left\{a_{1}, a_{2}\right\}\right),
$$

thus

$$
\begin{aligned}
& v_{i}^{*}\left[G^{\prime}\left(p\left(v_{i}^{*}+\delta, v_{-i}^{*}\right)\right)-G^{\prime}\left(p^{*}\right)\right]\left(v_{i}^{*}+\delta\right)-\delta \\
& \leq\left[v_{i}^{*}\left(v_{i}^{*}+\delta\right) \frac{2}{\left(\frac{a_{1}+a_{2}}{\varepsilon \min \left\{a_{1}, a_{2}\right\}}+3 \max \left\{a_{1}, a_{2}\right\}\right)^{2}}-1\right] \delta<0 .
\end{aligned}
$$

which finally proves (a).

Analogously, to prove (b), it is easy to show that

$$
\begin{aligned}
& v_{i}^{*}\left[G^{\prime}\left(p\left(v_{i}^{*}-\delta, v_{-i}^{*}\right)\right)-G^{\prime}\left(p^{*}\right)\right]\left(v_{i}^{*}-\delta\right)+\delta \\
& \geq\left[1-v_{i}^{*}\left(v_{i}^{*}-\delta\right) \frac{2}{\left(\frac{a_{1}+a_{2}}{\varepsilon \min \left\{a_{1}, a_{2}\right\}}+3 \max \left\{a_{1}, a_{2}\right\}\right)^{2}}\right] \delta>0 .
\end{aligned}
$$

The theorem has been proved. 


\section{References}

Allaz B, Vila JL (1993) Cournot competition, forward markets and efficiency. J Econ Theory 59:1-16

Başar T, Olsder GJ (1982) Dynamic non-cooperative game theory. Academic Press, New York

Ben-Akiva M, Bierlaire M, Burton D, Koutsopoulos HN, Mishalani R (2001) Network state estimation and prediction for real-time traffic management. Netw Spat Econ 1:293-318

Bowley AL (1924) The mathematical groundwork of economics. Oxford University Press, Oxford

Bresnahan TF (1981) Duopoly models with consistent conjectures. Am Econ Rev 71:934-945

Bulavsky VA (1997) Structure of demand and equilibrium in a model of oligopoly. Economics and Mathematical Methods (Ekonomiko-Matematicheskie Metody) 33:112-134. (in Russian)

Bulavsky VA, Kalashnikov VV (1994) One-parametric method to study equilibrium. Economics and Mathematical Methods (Ekonomiko-Matematicheskie Metody) 30:129-138. (in Russian)

Bulavsky VA, Kalashnikov VV (1995) Equilibrium in generalized Cournot and Stackelberg models. Economics and Mathematical Methods (Ekonomiko-Matematicheskie Metody) 31:164-176. (in Russian)

Cornes RC, Sandler T (1984) Easy riders, joint production, and public goods. Econ J 94:580-598

Driskill R, McCafferty S (1989) Dynamic duopoly with output adjustment costs in international markets. In: Feenstra E (ed) Trade policies for international competitiveness. University of Chicago Press, Chicago, pp 125-144

Fershtman C, Kamien M (1987) Dynamic duopolistic competition with sticky prices. Econometrica 55:1151-1164

Figuières C, Jean-Marie A, Quérou N, Tidball M (2004) Theory of conjectural variations. World Scientific, New Jersey/London/Singapore/Shanghai/Hong Kong/Taipei/Bangalore

Frisch R (1933) Monopole, polypole - La notion de force en économie. National Økonomisk Tidsskrift 71:241-259; reprinted (1951): monopoly, polypoly: the concept of force in the economy. International Economics Papers 1:23-36

Giocoli N (2005) The escape from conjectural variations: the consistency condition in duopoly theory from Bowley to Fellner. Cambridge J Econ 29:601-618

Isac G, Bulavsky VA, Kalashnikov VV (2002) Complementarity, equilibrium efficiency and economics. Kluwer Academic Publishers, Dordrecht/London/Boston

Kalashnikov VV, Bulavsky VA, Kalashnykova NI, Castillo FJ (2011) Mixed oligopoly with consistent conjectures. Eur J Oper Res 201:729-735

Kalashnikov VV, Bulavsky VA, Kalashnykova NI, López-Ramos F (2017) Consistent conjectures are optimal Cournot-Nash strategies in the meta-game. Optimization 66:2007-2024

Kalashnikov VV, Cordero E, Kalashnikov VV-Jr (2010) Cournot and Stackelberg equilibrium in mixed duopoly models. Optimization 59:689-706

Kalashnikov VV, Kemfert C, Kalashnikov VV-Jr (2009) Conjectural variations equilibrium in a mixed duopoly. Eur J Oper Res 192:717-729

Kalashnykova NI, Bulavsky VA, Kalashnikov VV (2012) Consistent conjectures as optimal Nash strategies in the upper level game. ICIC Express Letters 6:965-970

Kimbrough SO, Murphy FH, Smeers Y (2014) Extending Cournot: What does insight dissipate? Fox School of Business Research Paper No. 14-036; SSRN 2510537, https://papers.ssrn.com/sol3/papers. cfm?abstract_id=2510537. Accessed 29 July 2018

Kreps DM, Scheinkman JA (1983) Quantity precommitment and Bertrand competition yield Cournot outcomes. Bell J Econ 14:326-337

Kress D, Pesch E (2015) Competitive location and pricing on networks with random utilities. Netw Spat Econ 16:837-863

Laitner J (1980) "Rational” duopoly equilibria. Q J Econ 95:641-662

Lindh T (1992) The inconsistency of consistent conjectures. Coming back to Cournot. J Econ Behav Organ 18:69-90

Liu YF, Ni YX, Wu FF, Cai B (2007) Existence of uniqueness of consistent conjectural variation equilibrium in electricity markets. Int J Elec Power 29:455-461

Murphy FH, Smeers Y (2005) Generation capacity expansion in imperfectly competitive restructured electricity markets. Oper Res 53:646-661

Novshek W (1985) On the existence of Cournot equilibrium. Rev Econ Stud 52:85-98

Perry MK (1982) Oligopoly and consistent conjectural variations. Bell J Econ 13:197-205 
Sugden R (1985) Consistent conjectures and voluntary contributions to public goods: Why the conventional theory does not work. J Public Econ 27:117-124

Zerrahn A, Huppmann D (2017) Network expansion to mitigate market power. Netw Spat Econ 17:611644

Publisher's Note Springer Nature remains neutral with regard to jurisdictional claims in published maps and institutional affiliations.

\section{Affiliations}

\section{Viacheslav Kalashnikov 1,2,3 (D) . Nataliya Kalashnykova ${ }^{4}$ José G. Flores-Muñiz ${ }^{4}$}

Nataliya Kalashnykova

nkalash2009@gmail.com

José G. Flores-Muñiz

jose_guadalupe64@hotmail.com

1 Tecnologico de Monterrey (ITESM), Campus Monterrey, Av. Eugenio Garza Sada 2501 Sur, Monterrey, Nuevo León 64849, Mexico

2 Central Economics and Mathematics Institute (CEMI), Nakhimovsky pr. 47, Moscow 117418, Russia

3 Sumy State University (SumDU), Rimsky-Korsakov st. 2, Sumy, 40007, Ukraine

4 Universidad Autónoma de Nuevo León (UANL), Av. Universidad S/N, San Nicolás de los Garza, Nuevo León 66455, Mexico 